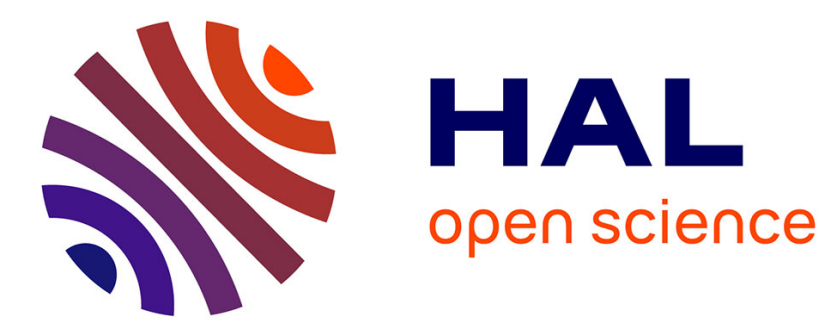

\title{
Low-temperature Transport Properties of the n-type Layered Homologous Compounds Bi8-xSbxSe7
}

Pavan Kumar Ventrapati, Shantanu Misra, Gaëlle Delaizir, Anne Dauscher, Bertrand Lenoir, Christophe Candolfi

\section{- To cite this version:}

Pavan Kumar Ventrapati, Shantanu Misra, Gaëlle Delaizir, Anne Dauscher, Bertrand Lenoir, et al.. Low-temperature Transport Properties of the n-type Layered Homologous Compounds Bi8xSbxSe7. Journal of Materials Chemistry C, 2020, 8 (40), pp.14037-14048. 10.1039/D0TC03935G . hal-03027391

\section{HAL Id: hal-03027391 \\ https://hal.science/hal-03027391}

Submitted on 27 Nov 2020

HAL is a multi-disciplinary open access archive for the deposit and dissemination of scientific research documents, whether they are published or not. The documents may come from teaching and research institutions in France or abroad, or from public or private research centers.
L'archive ouverte pluridisciplinaire HAL, est destinée au dépôt et à la diffusion de documents scientifiques de niveau recherche, publiés ou non, émanant des établissements d'enseignement et de recherche français ou étrangers, des laboratoires publics ou privés. 


\title{
Low-temperature Transport Properties of the $n$-type Layered Homologous Compounds $\mathrm{Bi}_{-x} \mathrm{Sb}_{x} \mathrm{Se}_{7}$
}

Pavan Kumar-Ventrapati ${ }^{1}$, Shantanu Misra ${ }^{1}$, Gaëlle Delaizir ${ }^{2}$, Anne Dauscher ${ }^{1}$, Bertrand Lenoir $^{1}$ and Christophe Candolfi ${ }^{1, *}$

${ }^{1}$ Institut Jean Lamour, UMR 7198 CNRS - Université de Lorraine, 2 allée André Guinier Campus ARTEM, 54000 Nancy, France

${ }^{2}$ Sciences des Procédés Céramique et de Traitement de Surface (SPCTS), UMR CNRS $7315-$ Univsersité de Limoges, Limoges, France

*Corresponding author: christophe.candolfi@univ-lorraine.fr

\begin{abstract}
Chalcogenide semiconductors and semimetals are a historical fertile class of materials for discovering novel compounds for energy conversion applications, such as photovoltaic cells or thermoelectric devices. Here, we report on a detailed investigation of the low-temperature transport properties $(5-300 \mathrm{~K})$ of the $n$-type series $\mathrm{Bi}_{8-x} \mathrm{Sb}_{x} \mathrm{Se}_{7}(0 \leq x \leq 2.4)$, the thermoelectric performances of which can be optimized around $300 \mathrm{~K}$. The complex layered crystal structure, built up by Bi bilayers and quintuple Bi-Se layers stacked perpendicular to the $c$ axis of the trigonal unit cell, gives rise to very low lattice thermal conductivity $\kappa_{L}$ values on the order of $0.6 \mathrm{~W} \mathrm{~m}^{-1} \mathrm{~K}^{-1}$ at $300 \mathrm{~K}$. Upon cooling, $\kappa_{L}(T)$ exhibits a crystalline-like behavior with a well-defined dielectric maximum. Despite $\mathrm{Sb}$ and $\mathrm{Bi}$ are isovalent, both the thermopower $\alpha$ and the electrical resistivity $\rho$ vary upon increasing the Sb content, possibly
\end{abstract}


due to variations in the nature and concentration of native defects. Our results indicate the existence of two substitutional ranges, in which the evolution of the electronic properties with $x$ are not equivalent. Combined with the poor ability of these materials to conduct heat, the high power factors, optimized for $x=1.6$, result in a maximum dimensionless thermoelectric figure of merit $Z T$ as high as 0.40 at $300 \mathrm{~K}$, making these compounds competitive among $n$ type thermoelectric chalcogenides for near-room-temperature thermoelectric applications.

\section{Introduction}

Thermoelectric materials offer an elegant and versatile way to directly convert heat into electrical power or generate a temperature difference when subjected to a current flow. ${ }^{1,2}$ Despite being the technology of choice to power deep-space probes and Mars rovers, thermoelectric devices have been so far limited to niche applications due to their low conversion efficiency. ${ }^{1,2}$ The efficiency with which energy conversion is realized is determined by the thermoelectric dimensionless figure of merit $Z T=\alpha^{2} T / \rho\left(\kappa_{L}+\kappa_{e}\right)$ where $T$ is the absolute temperature, $\alpha$ is the Seebeck coefficient or thermopower, $\rho$ is the electrical resistivity, $\kappa_{L}$ is the lattice thermal conductivity and $\kappa_{e}$ is the electronic thermal conductivity. ${ }^{1,2}$ The interdependence of $\alpha, \rho$ and $\kappa_{e}$ via the carrier concentration makes the optimization of the $Z T$ values a challenging undertaking. Band structure engineering, which aims to optimize the power factor $\alpha^{2} / \rho$ through band convergence or resonant levels, ${ }^{3-10}$ and searching for materials exhibiting very low $\kappa_{L}$ values, ${ }^{10-20}$ are the two general strategies pursued to mitigate these difficulties. Along this second line of research, cage-like structures and materials showing a high degree of lattice anharmonicity or structural complexity are prominent examples of compounds sought after in thermoelectricity. ${ }^{10-20}$ 
Despite significant theoretical and experimental efforts devoted over the last decades, most efficient thermoelectric materials discovered to date reach their peak $Z T$ values at high temperatures $(600-1200 \mathrm{~K}) .^{2}$ Only few examples of material exhibiting high $Z T$ values at or slightly above room temperature $(300-400 \mathrm{~K})$ are known. In addition to the conventional $(\mathrm{Bi}, \mathrm{Sb})_{2}(\mathrm{Te}, \mathrm{Se})_{3}$ alloys that remain the best materials for solid-state cooling around room temperature, ${ }^{1,2}$ novel $n$-type compounds such as $\mathrm{MgAgSb}$ or $\mathrm{Mg}_{3}(\mathrm{Bi}, \mathrm{Sb})_{2}$ have been recently reported. ${ }^{21-25}$ In these compounds, the peak $Z T$ values achieved (between 0.4 and 0.8 near 300 $\mathrm{K})$ rival those obtained in the state-of-the-art $n$-type $\mathrm{Bi}_{2} \mathrm{Te} 3$-based alloys. ${ }^{21-25}$ These high thermoelectric performances stem from the high degeneracy of the conduction bands that yield high thermopower values, combined with highly-anharmonic phonon branches that strongly limit the heat transport to values well below $1 \mathrm{~W} \mathrm{~m}^{-1} \mathrm{~K}^{-1}$ around $300 \mathrm{~K}^{21-25}$

Homologous compounds possess similar high structural complexity and significant chemical flexibility, making them another excellent research area to design novel thermoelectric materials. ${ }^{26-31}$ The crystal structure of these compounds is built from one or several types of slabs, the thicknesses and stacking sequence of which expand in one or several spatial directions by regular increments. The chemical composition of a homologous series can be rationalized by integer numbers, which can help to predict novel compounds. ${ }^{26}$ Several families of homologous compounds naturally exist as minerals, some of them, such as the cannizzarite, pavonite or lillianite, have been shown to exhibit interesting thermoelectric properties. $^{28-33}$

Among them, the tetradymite series stand out due to the fact that the above-mentioned $\mathrm{Bi}_{2} \mathrm{Te}_{3}$-based compounds belong to this broad family of compounds. In the Bi-Se sub-system, thirteen binary compounds with varying Bi-to-Se ratio have been identified so far, spanning the phase space from elemental semi-metallic $\mathrm{Bi}$ to semiconducting $\mathrm{Bi}_{2} \mathrm{Se}_{3} .{ }^{34-38}$ The continuous insertion of $\mathrm{Bi}$ into $\mathrm{Bi}_{2} \mathrm{Se}_{3}$ creates a homologous series of layered compounds of 
general chemical formula $\left(\mathrm{Bi}_{2}\right)_{n}\left(\mathrm{Bi}_{2} \mathrm{Se}_{3}\right)_{m}$ with $n$ and $m$ being integers. The crystal structure of each of these compounds is defined by a specific sequence of two-atom-thick ( $\left.\mathrm{Bi}_{2}\right)$ and quintuple layers $\left(\mathrm{Bi}_{2} \mathrm{Se}_{3}\right)$ separated by van der Waals gaps. Varying $n$ and $m$ produces both commensurate and incommensurate stackings. Because the total number of layers pet unit cell is a multiple of 3 , both trigonal and rhombohedral structures are formed described in $P \overline{3} m 1$ or $R \overline{3} m$ space group, respectively. ${ }^{34-38}$ An unified description of these crystal structures was developed based on a hexagonal subcell modulated along the $c$ axis with a modulation vector $\vec{q}=\gamma \vec{c}^{*} \cdot{ }^{37}$ Within this approach, the constant $\gamma=3 \frac{(3 m+n)}{N}$ varies with the chemical composition according to the above-mentioned $n$ and $m$ integers that defines the rhombohedral or trigonal stacking sequence determined by $3 m+n$ and the total number of atomic layers in the stacking sequence $N=5 m+2 n \cdot{ }^{37}$ When $\gamma$ is a rational number, the modulation is commensurate while incommensurate superstructures appear for non-rational values. $^{34-38}$

Based on electronic band structure calculations, ${ }^{38}$ the addition of zero-valent $\mathrm{Bi}_{2}$ layers in $\mathrm{Bi}_{2} \mathrm{Se}_{3}$ was shown to tend to close the electronic band gap of $\mathrm{Bi}_{2} \mathrm{Se}_{3}$, producing a series of semi-metallic compounds. In agreement with these predictions, recent studies of the thermoelectric properties of the binaries $\mathrm{BiSe}, \mathrm{Bi}_{8} \mathrm{Se}_{9}$ and $\mathrm{Bi}_{8} \mathrm{Se}_{7}$ evidenced that these compounds behave as narrow-band-gap $n$-type semiconductors. ${ }^{39-41}$ Remarkably, all these compounds exhibit $\kappa_{L}$ values of around $0.5 \mathrm{~W} \mathrm{~m}^{-1} \mathrm{~K}^{-1}$ at $600 \mathrm{~K}$ that are significantly lower than those of $\mathrm{Bi}_{2} \mathrm{Se}_{3}$. In $\mathrm{Bi}_{8} \mathrm{Se}_{7}$, the co-substitution of $\mathrm{Sb}$ and $\mathrm{Te}$ for $\mathrm{Bi}$ and $\mathrm{Se}$, respectively, led to a maximum $Z T$ values of 0.7 at $420 \mathrm{~K},{ }^{41}$ comparable to that achieved in BiSe with a peak $Z T$ value of 0.8 at $425 \mathrm{~K} .{ }^{39}$

Here, we report on a detailed investigation of the low-transport properties $(5-300 \mathrm{~K})$ of the series $\mathrm{Bi}_{8-x} \mathrm{Sb}_{x} \mathrm{Se}_{7}$ for $0.0 \leq x \leq 2.4$ in order to explore the basic mechanisms that govern their electronic and thermal properties. In agreement with a prior study on this series ${ }^{41}$ our 
results confirm very low lattice thermal conductivity, on the order of $0.6 \mathrm{~W} \mathrm{~m}^{-1} \mathrm{~K}^{-1}$ near 300 $\mathrm{K}$, similar to those reported for the binaries BiSe and BisSe $9 .{ }^{39,40}$ However, the evolution of the electronic properties with $x$ appears more complex than previously reported, suggesting an important role played by native defects, similarly to what has been observed in other relevant chalcogenide compounds for thermoelectric applications.

\section{Experimental Section}

\subsection{Synthesis and sample characterization}

All handling of elements and powders were carried out in an argon-filled, dry glove box. Prior to use, elemental $\mathrm{Bi}(99.999 \%), \mathrm{Sb}$ and $\mathrm{Te}(99.999 \%)$ were purified to remove possible traces of oxides. To this end, each element was loaded in a pre-evacuated silica tube, sealed under inert atmosphere, heated above its melting point in a rocking furnace for $2 \mathrm{~h}$ and finally quenched in room-temperature water. The surfaces of the obtained ingots were carefully polished in the glove box before being hand ground into fine powders. Polycrystalline $\mathrm{Bi}$ ${ }_{x} \mathrm{Sb}_{x} \mathrm{Se}_{7}$ samples with $x=0.0,0.8,1.2,1.6,2.0$ and 2.4 were subsequently prepared from a mixture of stoichiometric amounts of purified $\mathrm{Bi}, \mathrm{Sb}$ and $\mathrm{Te}$ powders. These mixtures were loaded in pre-evacuated silica tubes and sealed under inert atmosphere. The tubes were placed in a rocking furnace and heated to $650^{\circ} \mathrm{C}$, that is, above the melting point of the binary $\mathrm{Bi}_{8} \mathrm{Se}_{7}$ according to the Bi-Se binary phase diagram. ${ }^{42}$ After maintaining this temperature for 2 days, the tubes were quenched in room-temperature water. The obtained ingots were hand ground into fine powders that were cold-pressed into cylindrical pellets. These pellets were sealed in silica tubes under inert atmosphere, annealed at $450^{\circ} \mathrm{C}$ for 15 days, before being eventually quenched in room-temperature water. Of note, this long-term annealing is an important 
requirement to achieve phase-pure polycrystalline samples of compounds belonging to the homologous series $\left(\mathrm{Bi}_{2}\right)_{n}\left(\mathrm{Bi}_{2} \mathrm{Se}_{3}\right)_{m}$ due to the numerous phases with close chemical compositions that exist in the phase diagram. ${ }^{42}$ Traces of other phases may still be present in short-term annealed samples, which may influence the measured transport properties. The annealed pellets were hand ground into fine powders that were consolidated by spark plasma sintering in a graphite die at $450^{\circ} \mathrm{C}$ under a uniaxial pressure of $65 \mathrm{MPa}$ for $10 \mathrm{~min}$. The density of the consolidated cylindrical pellets was determined from weight and geometrical dimensions. For all samples, the density was higher than $95 \%$ of the theoretical density.

The crystal structure and phase purity of the final compounds were verified by powder X-ray diffraction (PXRD) performed at $300 \mathrm{~K}$ using a D8 Bruker Advance diffractometer equipped with a LynxEye detector $\left(\mathrm{Cu} K \alpha_{1}\right.$ radiation, $\left.\lambda=1.54056 \AA\right)$. Because of the strongly anisotropic crystal structure of these compounds, the lattice parameters were inferred from refinements of the PXRD patterns in profile matching mode using the FullProf software. ${ }^{43}$

SEM experiments were performed on polished bulk pieces cut from the consolidated ingots using a Quanta FEG 650 (FEI) with energy-dispersive X-ray spectroscopy (EDXS). Images in backscattered electron mode (BSE) and elemental X-ray maps were taken to assess phase purity and determine the spatial distribution of $\mathrm{Bi}, \mathrm{Sb}$ and $\mathrm{Se}$.

\subsection{Optical absorption measurements}

Room-temperature optical measurements were performed on powdered samples using diffuse reflectance spectroscopy on a Thermo Scientific Nicolet 6700 FTIR spectrophotometer equipped with an integrated sphere. Raw reflectance data $R$ were used to estimate the absorption coefficient using the Kubelka-Munk relation $F(R)=(1-R)^{2} / 2 R .^{44}$ The data were then plotted as $(F(R) \hbar \omega)^{n}$ versus $\hbar \omega$, where $\hbar \omega$ is the incoming photon energy, to 
estimate the transition onset energies corresponding to the optical band gap $E_{g}$. Both direct ( $n$ $=2)$ and indirect $(n=1 / 2)$ extrapolations of the absorption edge to zero were considered (see Figure S1 in the ESI file).

\subsection{Transport property measurements}

Due to the layered crystal structure of the samples, the transport properties were measured on samples cut perpendicular and parallel to the pressing direction with a diamond-wire saw. Electrical resistivity, thermopower and thermal conductivity were measured simultaneously on bar-shaped samples (typical dimensions of $\left.2 \times 2 \times 8 \mathrm{~mm}^{3}\right)$ at low temperatures $(5-300 \mathrm{~K})$ using the thermal transport option of a physical property measurement system (PPMS, Quantum Design). Good electrical and thermal contacts were realized by gluing copper bars with a minute amount of conductive silver epoxy. Hall effect measurements were performed on the same bar-shaped samples using the AC transport option of the PPMS. A five-probe configuration was used by brazing five copper wires onto the samples using a low-meltingpoint braze. The transverse electrical resistivity $\rho_{x y}$ was measured at selected temperatures between 5 and $300 \mathrm{~K}$ under magnetic fields $\mu_{0} H$ ranging between -1 and $+1 \mathrm{~T}$. Possible magnetoresistive contributions were dismissed by considering the antisymmetric part of $\rho_{x y}$

under magnetic field reversal following the formula $\left[\rho_{x y}\left(+\mu_{0} H\right)-\rho_{x y}\left(-\mu_{0} H\right)\right] / 2$. The Hall electron concentrations $n_{H}$ and mobilities $\mu_{H}$ were inferred by the single-band formulas $n_{H}=$ $-1 / R_{H} e$ and $\mu_{H}=R_{H} / \rho$ where $R_{H}$ is the Hall coefficient and $e$ is the elementary charge. Of note, the strongly anisotropic crystal structure of these compounds implies galvanomagnetic properties to be described by a tensor containing several independent components, the number of which is determined by the symmetry of the crystal lattice. ${ }^{45}$ The $R_{H}$ values measured parallel and perpendicular to the pressing direction in polycrystalline samples correspond to a 
different mixing of these components. A strong anisotropy in $R_{H}$ makes the above-mentioned single-band formulas relating $n_{H}$ and $\mu_{H}$ to $R_{H}$ incorrect. In order to estimate the degree of anisotropy in the $R_{H}$ values for the present series of samples, measurements on samples cut parallel and perpendicular were realized. As shown in Figure S2 in the ESI file, the anisotropy in the binary compound between both directions is negligible, which justifies the approximation used herein. For sake of completeness, high-temperature thermoelectric measurements have been also performed, the details of which can be found in the ESI file. The experimental uncertainty associated with these measurements are estimated to be $5 \%$ for all transport properties at low temperatures, 5\% at high temperatures for the electrical resistivity and thermopower and $10 \%$ for the thermal conductivity. The combined experimental uncertainty in the determination of the $Z T$ values is estimated to be $17 \% .{ }^{46} \mathrm{An}$ overall good match near room temperature is observed between the low- and hightemperature electrical resistivity and thermopower measurements. In contrast, the larger difference observed in the thermal conductivity is due to the thermal radiations that accompany the low-temperature thermal conductivity measurements and tend to increase the measured values above $\sim 200 \mathrm{~K}$.

\section{Results and discussion}

\subsection{Crystal structure and phase purity}

$\mathrm{Bi}_{8} \mathrm{Se}_{7}$ adopts a complex trigonal layered crystal structure described in the space group $P \overline{3} m 1 .{ }^{47}$ The $\mathrm{Bi}$ and $\mathrm{Se}$ atoms are distributed over twelve and eleven independent crystallographic sites, respectively. The crystal structure is formed by two distinct types of layers, stacked along the $c$ axis, built up by $\mathrm{Bi}_{2}$ zigzag chains separating tetradymite-like 
$\mathrm{Bi}_{2} \mathrm{Se}_{3}$ quintuple layers (Figure 1). These quintuple layers, similar to those observed in the $\operatorname{Bi}_{2} X_{3}(X=\mathrm{S}, \mathrm{Se}$ and $\mathrm{Te})$ thermoelectric materials, can be viewed as chains of edge-sharing $\mathrm{BiSe}_{6}$ octahedra. In tetradymite-like compounds, should they be natural or synthetic, these types of layers are described using the nomenclature ' 2 ' and ' 5 ', respectively, that correspond to the slab thickness. Following this description, the long-periodic stacking sequence of these layers in $\mathrm{Bi}_{8} \mathrm{Se}$ c can be written as 2525525255252 giving rise to the developed chemical formula $\left(\mathrm{Bi}_{2}\right)_{n}\left(\mathrm{Bi}_{2} \mathrm{Se}_{3}\right)_{m}$ with $n=5$ and $m=7$. In this series, the lattice parameter $c$ can be predicted from the integers $n$ and $m$ following the formula $c_{\text {predicted }}=\frac{1}{3}\left[n c_{B i}+m c_{B i_{2} S e_{3}}\right]$ where $c_{B i}$ and $c_{B i_{2} S e_{3}}$ are the lattice parameters of elemental $\mathrm{Bi}$ and $\mathrm{Bi}_{2} \mathrm{Se}_{3}$, respectively. ${ }^{48}$ Using $c_{B i}=11.589 \AA$ and $c_{B i_{2} S e_{3}}=28.70 \AA \AA^{1,2,48}$ this formula predicts a lattice parameter $c$ of $86.28 \AA$ for $\mathrm{Bi}_{8} \mathrm{Se}_{7}$, which is indeed close to our refined experimental value of $86.04(1) \AA$.

Regardless of the Sb content, all the reflections observed in the PXRD patterns (Figure 2a) can be well indexed to the $\mathrm{Bi}_{8} \mathrm{Se}_{7}$-type phase indicating that all samples crystallize with the same crystal structure. No evident additional reflections are observed suggesting the absence of secondary phases within the detection limit of this technique. The comparison between the theoretical and experimental X-ray diffraction patterns clearly evidence the strong renormalization of the observed reflections, reflecting the anisotropic crystal structure of these compounds, which inevitably leads to the tendency of the crystallites to show some preferred orientation in polycrystalline samples. The successful insertion of Sb into the crystal structure is further confirmed by the systematic shift of some reflections with increasing the $\mathrm{Sb}$ content (Figure 2b), indicative of a decrease in the unit cell volume $V$. The linear shrinkage of $V$ with increasing $x$ (Figure 3a) is consistent with the smaller covalent radius of $\mathrm{Sb}(139 \mathrm{pm})$ with respect to $\mathrm{Bi}(148 \mathrm{pm})$, further confirming the successful substitution of $\mathrm{Sb}$ for $\mathrm{Bi}$ up to $x=2.4$, which is below the solubility limit of $\mathrm{Sb}$ in $\mathrm{Bi}_{8} \mathrm{Se}$ 7 determined to be $x=$ 3.6. ${ }^{41}$ However, unlike previous results reporting linear decreases for both lattice 
parameters, ${ }^{41}$ only $a$ follows a linear trend, while the concomitant contraction of the $c$ axis evolves non-linearly with increasing $x$ (Figure 3b). Below $x=1.6, c$ exhibits a non-linear evolution with a faster decrease above $x=0.8$. In contrast, for $x \geq 1.6, c$ varies quasi-linearly with increasing the $\mathrm{Sb}$ concentration up to $x=2.4$. These two distinct trends, that will manifest on the transport properties discussed below, might be related to the type of layers, $\mathrm{Bi} 2$ or $\mathrm{Bi}_{2} \mathrm{Se}_{3}$, into which Sb substitutes for $\mathrm{Bi}$.

BSE images and the corresponding X-ray maps are shown in Figure 4 for the illustrative Sb-containing sample $x=1.6$. For all samples, both Bi and Se are homogeneously distributed within the samples with no indications of spatial variations. The anisotropy in the microstructure of these samples was further investigated on freshly-fractured surfaces of the end-member sample $x=0.0$ (Figure 5), representative of the microstructure observed across the entire series. Both samples exhibit grains arranged in a layered manner that tend to be aligned perpendicular to the SPS pressing direction. Although these different layered areas are not all oriented along the same direction, they are nevertheless expected to give rise to anisotropic transport properties when measured along and perpendicular to the pressing direction.

\subsection{Optical and Electronic Transport Properties}

Figure 6 shows the optical absorption spectra measured for the $x=0.0,1.2$ and 2.4 samples, plotted assuming direct optical transitions. The qualitative conclusions drawn hereafter remain unchanged assuming indirect optical transitions (see Figure S1 in ESI). For all samples, the spectra show a slow, continuous rise for energies above the minimum energy measured of 50 meV. A faster rise is observed above $\sim 0.42 \mathrm{eV}$, a threshold that hardly depend on the $\mathrm{Sb}$ concentration. Based on electronic band structure calculations available in topological 
material databases, ${ }^{49}$ in agreement with recent calculations, ${ }^{41} \mathrm{Bi}_{8} \mathrm{Se}_{7}$ has been identified as either a semiconductor with a narrow band gap, unresolved in these calculations, or a semimetal, depending on whether spin-orbit coupling is included or not, respectively. In both cases, several possible interband transitions of small amplitude are present, which may explain the slow but continuous rise of the absorption signal observed below $0.40 \mathrm{eV}$. This transition possibly corresponds to an indirect interband transition from one of the low-laying valence bands to the conduction bands. Considering the calculations performed including spin-orbit coupling, the slow rise at low energies may be interpreted as transitions across a small band gap on the order of $100 \mathrm{meV}$ for the three samples.

The temperature dependence of the electrical resistivity $\rho$ and thermopower $\alpha$ are shown in Figures $7 \mathrm{a}$ and $7 \mathrm{~b}$, respectively. For sake of clarity, and because the anisotropy in the transport properties hardly evolve in temperature or with $x$, we only present data measured parallel to the pressing direction, the direction along which the highest thermoelectric performances have been obtained. The main conclusions drawn from these data would not be affected by considering the values measured in the perpendicular direction, which can be found in the ESI file (Figures S3 and S4).

For $\mathrm{Bi}_{8} \mathrm{Se}_{7}, \rho$ increases with increasing temperature up to around $300 \mathrm{~K}$, where a maximum is reached (see Figure S5 in ESI for measurements up to $700 \mathrm{~K}$ ). This temperature dependence and the relatively low $\rho$ values are characteristic of compensated semiconductors observed in both narrow-band-gap semiconductors and semi-metals. While these results are consistent with those obtained for BiSe and for several compounds in the closely-related homologous series $\left(\mathrm{Bi}_{2}\right)_{n}\left(\mathrm{Bi}_{2} \mathrm{Te}_{3}\right)_{m},{ }^{39,48,50}$ these $\rho(T)$ data differ from those reported for $\mathrm{Bi}_{8} \mathrm{Se}_{9}$ for which, a metallic character is maintained up to $700 \mathrm{~K}$. The comparison between these three compounds shows that the details of the electronic band structure and notably, the possible narrow band gap is sensitive to the stacking sequence. ${ }^{38}$ 
The substitution of $\mathrm{Sb}$ for Bi does not significantly modify the temperature dependence that characterizes the binary compound, which persists across the entire Sb concentration range. However, increasing the $\mathrm{Sb}$ content tends to increase both the $\rho$ values and the lowtemperature slope $\partial \rho / \partial T$. Unlike prior results for which a monotonic variation with $x$ has been observed, ${ }^{41}$ the evolution of $\rho(T)$ with $x$ can be divided into two distinct concentration ranges in which, the variations in $\rho(T)$ are not strictly equivalent. In the first compositional range extending from $x=0.0$ up to $x=1.2$, the monotonic increase in $\rho$ with $x$ is accompanied by a shift in the maximum in $\rho(T)$ from 300 to $270 \mathrm{~K}$ on going from $x=0.0$ to $x=1.2$. The second concentration range is then reached upon crossing a critical $x$ value lying between $x=$ 1.2 and $x=1.6$. Increasing $x$ to $x=1.6$ leads to a shift of the maximum (reached at $360 \mathrm{~K}$, Figure S5 in ESI) but without a further increase in the $\rho$ values which remain practically equivalent to those measured in the $x=1.2$ sample. In contrast to the first compositional range, further increasing the $\mathrm{Sb}$ content to $x=2.4$ does not change the temperature at which the maximum is attained. However, meanwhile, both the $\rho$ values and the slope $\partial \rho / \partial T$ increase with $x$. The existence of a Sb concentration limit delineating two distinct transport behaviors is consistent with the aforementioned variations in the lattice parameters with $x$ and further suggests that more significant modifications in the electronic band structure occur at high Sb contents. As we shall see below, this scenario is supported by the analysis of the thermopower dependence on the carrier concentration.

Despite the strongly anisotropic crystal structure of $\mathrm{Bi}_{8} \mathrm{Se}_{7}$, the anisotropy remains moderate over the entire temperature range with a nearly temperature-independent anisotropy ratio $\rho_{\|} / \rho_{\perp}$ of 1.3 between the parallel and perpendicular directions. Upon substituting Sb for $\mathrm{Bi}$, the anisotropy ratio varies from 1.1 for $x=1.6$ up to 1.4 for $x=0.8$. The fact that no clear trend in the variations in $\rho_{\|} / \rho_{\perp}$ with $x$ is observed may be attributed to microstructural 
aspects, with the relative orientation of the crystallites that tend to align perpendicular to the pressing direction possibly slightly varying from sample to sample.

The thermopower values $\alpha$ are negative across the entire temperature and $x$ ranges (Figure $7 b$ ), indicative of electrons as the dominant charge carriers in the series $\mathrm{Bi}_{8-x} \mathrm{Sb}_{x} \mathrm{Se}_{7}$. For all samples, $\alpha$ increases in absolute values with increasing temperature up to about $250 \mathrm{~K}$ where $\alpha(T)$ tends to level off, reaches a maximum around $300 \mathrm{~K}$ (see Figure S5 in ESI). While the relatively low $\alpha$ values of the $x=0.0$ sample are consistent with its degenerate nature, increasing the Sb content leads to a mark increase in $\alpha$ from $-75 \mu \mathrm{V} \mathrm{K}^{-1}$ for $x=0.0$ to $-145 \mu \mathrm{V} \mathrm{K}{ }^{-1}$ for $x=2.4$ at $300 \mathrm{~K}$.

The $n$-type electronic properties observed in the $\mathrm{BiSe}, \mathrm{Bi}_{8} \mathrm{Se}_{9}, \mathrm{Bi}_{2} \mathrm{Se}_{3}$ and $\mathrm{Bi}_{8} \mathrm{Se}_{7}$ compounds ${ }^{2,39-41}$ contrasts with the homologous series $\left(\mathrm{Bi}_{2}\right)_{n}\left(\mathrm{Bi}_{2} \mathrm{Te}_{3}\right)_{m}$ in which, ${ }^{48}$ both $n$-type and $p$-type compounds were reported, without any clear correlation between the $n$ and $m$ values and the sign of $\alpha$. The fact that undoped $\mathrm{Bi}_{2} \mathrm{Se}_{3}$ is naturally $n$-type has been correlated to the presence of a high concentration of Se vacancies, each of them acting as double donors. ${ }^{2,51}$ The prevalence of $n$-type behavior observed so far in the homologous series $\left(\mathrm{Bi}_{2}\right)_{n}\left(\mathrm{Bi}_{2} \mathrm{Se}_{3}\right)_{m}$ suggests a similar origin.

The anisotropy in the $\alpha$ values evolves both with $x$ and $T$ (Figures S4a and S4b in ESI). Of note, $\alpha$ is generally defined by a tensor in anisotropic crystals, possibly giving rise to strongly-direction dependent $\alpha(T)$, as in single crystals of elemental $\mathrm{Bi}$, which is a canonical example of such a behavior. ${ }^{52}$ Although this anisotropy is often reduced in polycrystalline compounds, $\alpha$ may nevertheless still show some degree of anisotropy between measurements performed parallel and perpendicular to the pressing direction. The weak anisotropy observed below $200 \mathrm{~K}$ in the $x=0.0$ sample becomes more significant upon warming to $300 \mathrm{~K}$. Above this temperature, the anisotropy ratio $\alpha_{\|} / \alpha_{\perp}$ is practically temperature-independent. Increasing $x$ leads to significant decrease in $\alpha_{\|} / \alpha_{\perp}$, which becomes negligible below $300 \mathrm{~K}$ in 
the $x=2.4$ sample. The difference in the $\alpha$ values between the parallel and perpendicular directions may further reflect the asymmetry between the anisotropic electron and hole density-of-states effective masses due to different conduction and valence band dispersions. The lower anisotropy ratio observed for samples within the range $1.6 \leq x \leq 2.4$ suggests possible variations in the density-of-states effective masses with $x$, corroborating the two distinct trends observed in $\rho(T)$.

The maxima characterizing both $\rho(T)$ and $\alpha(T)$ near $300 \mathrm{~K}$ suggest that all samples experience minority carrier effects above this temperature. The thermal excitation of holes in the present case is expected to result in an increase in the carrier concentration, explaining the decrease in $\rho(T)$ at high temperatures (Figure S5 in ESI). In addition, because the contribution of electrons and holes to $\alpha$ are of opposite sign, this mechanism also consistently explains the rapid decrease observed in $\alpha(T)$. The fact that the maximum in $\alpha(T)$ weakly depends on the Sb concentration suggests that the possible band gap in the electronic band structure of $\mathrm{Bi}_{8} \mathrm{Se}_{7}$ is not strongly affected by this substitution. An estimate of the band gap width $E_{g}$ can be inferred using the Goldsmid-Sharp formula $E_{g}=2 e \alpha_{\max } T_{\max }$ that relates $E_{g}$ to the maximum thermopower value $\alpha_{\max }$ reached at $T_{\max } \cdot{ }^{53}$ In the present case, this relation yields $E_{g}$ values that slightly evolve from $48 \mathrm{meV}$ in the $x=0.0$ sample to $91 \mathrm{meV}$ in the $x=$ 2.4 sample. These small $E_{g}$ values, similar to that estimated in BiSe (85 meV), are consistent with the predicted tendency towards closing the band gap of $\mathrm{Bi}_{2} \mathrm{Se}_{3}$ upon adding $\mathrm{Bi}_{2}$ layers. ${ }^{38}$ These $E_{g}$ values should nevertheless be taken with some caution, due to the fact that the Goldsmid-Sharp relation is in principle only strictly applicable to non-degenerate samples.

Due to the significant increase in $\alpha$ with increasing the $\mathrm{Sb}$ concentration, the power factor $\alpha^{2} / \rho$ follows a similar trend. The best compromise between high $\alpha$ and low $\rho$ values is achieved in the $x=1.6$ sample with a peak power factor of $1.14 \mathrm{~mW} \mathrm{~m}^{-1} \mathrm{~K}^{-2}$ at $320 \mathrm{~K}$ in the parallel direction (see Figure S5 in ESI). This optimized value outperforms those achieved at 
the same temperature in $\mathrm{Sb}$-substituted $\mathrm{BiSe}$ or $\mathrm{Bi}_{8} \mathrm{Se}_{9}$ but remains lower than that achieved in pristine $\mathrm{Bi}_{2} \mathrm{Se}_{3}{ }^{2,39-41}$

The temperature and compositional dependence of the electron concentration $n_{H}$, shown in Figure 8a, are consistent with the main traits observed in both $\rho(T)$ and $\alpha(T)$. For $0.0 \leq x \leq$ $1.2, n_{H}$ shows a similar temperature dependence characterized by a plateau below about $50 \mathrm{~K}$ followed by an increase in $n_{H}$ with increasing temperature suggestive of a thermally-activated behavior. For these three samples, the $n_{H}$ value measured at $5 \mathrm{~K}$ nearly doubles at $300 \mathrm{~K}$. On further increasing $x$ to $x=1.6, n_{H}$ still increases with temperature, albeit with a weaker slope compared to the other samples, yielding an overall increase of 35\% upon warming from 5 to $300 \mathrm{~K}$. These temperature dependences are consistent with either a semi-metallic state or the presence of very narrow band gaps as suggested by the optical absorption spectra and the near-room-temperature maxima in $\rho(T)$ and $\alpha(T)$. These data further show that $n_{H}$ evolves in a non-linear manner with $x$. After an initial decrease in $n_{H}$ from $1.7 \times 10^{20} \mathrm{~cm}^{-3}$ for $x=0.0$ to $6.5 \times 10^{19} \mathrm{~cm}^{-3}$ for $x=2.4$ at $50 \mathrm{~K}, n_{H}$ increases to $1.2 \times 10^{20} \mathrm{~cm}^{-3}$ for $x=1.6$. Further increasing the Sb content only slightly influences $n_{H}$ that decreases to $1.0 \times 10^{20} \mathrm{~cm}^{-3}$ at $50 \mathrm{~K}$ in the $x=$ 2.4 sample.

The non-linear evolution of $n_{H}$ with $x$ bears some similarities with the variations in the electron concentration with the $\mathrm{Sb}$ content observed in the series $\mathrm{Bi}_{2-x} \mathrm{Sb}_{x} \mathrm{Se}_{3} .{ }^{54-56} \mathrm{Because} \mathrm{Sb}$ is isovalent with $\mathrm{Bi}$, the variations in the electron density were ascribed to the influence of $\mathrm{Sb}$ on the type and concentration of defects that characterize tetradymite compounds. The concentration of $\mathrm{Se}$ vacancies that prevail in $\mathrm{Bi}_{2} \mathrm{Se}_{3}$ decreases upon increasing the $\mathrm{Sb}$ concentration up to about $x=0.1$, resulting in lowered electron concentrations. ${ }^{57-59}$ For higher Sb concentrations, however, the weaker decrease in $n_{H}$ with $x$ was suggested to be a signature of either a decrease in donor-like Bise and $\mathrm{Se}_{\mathrm{Bi}}$ antisite defects or the formation of tripleacceptor Bi vacancies. ${ }^{60,61}$ In the present series $\mathrm{Bi}_{8-x} \mathrm{Sb}_{x} \mathrm{Se}_{7}$, the initial decrease in $n_{H}$ up to $x=$ 
1.2 mimics the behavior observed in $\mathrm{Bi}_{2-x} \mathrm{Sb}_{x} \mathrm{Se}_{3}$ and thus, might be related to a similar decrease in the concentration of Se vacancies. The significantly weaker dependence of $n_{H}$ on $x$ for $1.6 \leq x \leq 2.4$ might also be due to a further slight decrease in the concentration of Se vacancies or the formation of acceptor-like defects. Compared to $\mathrm{Bi}_{2-x} \mathrm{Sb}_{x} \mathrm{Se}_{3}$, the presence of additional $\mathrm{Bi}_{2}$ layers in $\mathrm{Bi}_{8-x} \mathrm{Sb}_{x} \mathrm{Se}_{7}$ adds another degree of complexity owing to the fact that $\mathrm{Sb}$ may substitute for $\mathrm{Bi}$ in both types of layers. Moreover, the substitution scheme might depend on $x$, that is, $\mathrm{Sb}$ might first enter the $\mathrm{Bi}_{2} \mathrm{Se}_{3}$ layers for low $x$ values followed by insertion in the $\mathrm{Bi}_{2}$ layers at higher $\mathrm{Sb}$ contents, or vice versa. A sequential substitution might explain alternatively the sudden increase in $n_{H}$ upon further increasing $x$ from $x=1.2$ to $x=$ 1.6. We note that recent formation energy calculations have suggested a rather random distribution of $\mathrm{Sb}$ in both types of layers. ${ }^{41}$ However, given the high number of inequivalent crystallographic Bi sites, further calculations, in addition to spectroscopic techniques probing the local atomic environment of $\mathrm{Bi}$ and $\mathrm{Se}$, seem necessary to clarify these aspects. In addition, such calculations would be helpful in determining both the dominant type of defects in $\mathrm{Bi}_{8} \mathrm{Se}_{7}$ and the exact role played by $\mathrm{Sb}$ on their nature and concentration.

The temperature dependence of the Hall mobility $\mu_{H}$ (Figure $8 \mathrm{~b}$ ) shows that the $\mathrm{Sb}$ content does not significantly influence the $\mu_{H}$ values and the electron scattering mechanisms. Similar across the entire $\mathrm{Sb}$ concentration range, $\mu_{H}(T)$ is characterized by constant values below $30 \mathrm{~K}$, suggestive of electrons being scattered by neutral impurities. Above this temperature, the $\mu_{H}$ values start to decrease with increasing $T$ up to about $200 \mathrm{~K}$ following a $T^{-3 / 2}$ law indicative of acoustic phonon scattering. While this mechanism likely remains the dominant source of electron diffusion in these samples below $300 \mathrm{~K}$, the $\mu_{H}$ values decrease with a slope steeper than $-3 / 2$ above $200 \mathrm{~K}$, likely due to the onset of minority carrier activation. As $\rho$ and $\alpha, \mu_{H}$ also evolves non-linearly with $x$, with values ranging between 70 and $95 \mathrm{~cm}^{2} \mathrm{~V}^{-1} \mathrm{~s}^{-1}$ at $5 \mathrm{~K}$ and between 25 and $40 \mathrm{~cm}^{2} \mathrm{~V}^{-1} \mathrm{~s}^{-1}$ at $300 \mathrm{~K}$ for the $x=0.8$ and $x=$ 
0.0 samples, respectively. For $x=0.8, \mu_{H}$ first decreases due to enhanced point defect scattering induced by the substitution of $\mathrm{Sb}$ for Bi. However, an additional increase in the $\mathrm{Sb}$ content does not further degrade the electron mobility, the values of which remain framed by the values of the $x=0.0$ and 0.8 samples. While the absence of further decrease in $\mu_{H}$ with increasing the disorder might be related to microstructural effects, increasing disorder is not necessarily at the expense of high carrier mobility, as highlighted for instance in the series of homologous compounds $(\mathrm{PbSe})_{5}\left(\mathrm{Bi}_{2} \mathrm{Se}_{3}\right)_{3 m}(m=1,2$ and 3$)$ or in the Zintl phase $\mathrm{Ca}_{9} \mathrm{Zn}_{4+x} \mathrm{Sb}_{9},{ }^{62,63}$ for which an increase in the complexity of the unit cell is not accompanied by a strong reduction in the charge carrier mobility.

In order to model the evolution of the electrical transport in the series $\mathrm{Bi}_{8-x} \mathrm{Sb}_{x} \mathrm{Se}_{7}$, the Ioffe-Pisarenko curve, which describes the variations in the thermopower with the carrier concentration, was calculated. Due to thermally-activated behavior evidenced by the $n_{H}(T)$ data above $50 \mathrm{~K}$ and the onset of minority carrier effects above $200 \mathrm{~K}$, we have restricted this analysis to $50 \mathrm{~K}$. The $\alpha\left(n_{H}\right)$ data, shown in Figure 9, further underline the two distinct regimes revealed by the transport data on crossing a critical Sb concentration lying between $x$ $=1.2$ and 1.6. The variations in each concentration range can be well described by a singleparabolic band model (SPB) with neutral impurity scattering that dominates below $50 \mathrm{~K} .{ }^{64}$ The SPB model yields a density-of-states effective mass $m_{D O S}^{*}$ of $1.1 m_{0}$ for $0.0 \leq x \leq 1.2$ that increases to $1.3 m_{0}$ for $1.6 \leq x \leq 2.4$, lower than those estimated at $425 \mathrm{~K}\left(m_{D O S}^{*} \approx 1.6 m_{0}\right),{ }^{41}$ that is, in the bipolar regime (see Figures S3 to S5 in ESI), which is not taken into account in the SPB model. The difference in $m_{D O S}^{*}$ between the two compositional ranges supports the idea that increasing the $\mathrm{Sb}$ content beyond $x=1.2$ is accompanied by a non-rigid-like evolution of the electronic band structure. 


\subsection{Thermal Transport Properties}

Figure 10a shows the temperature dependence of the total thermal conductivity $\kappa$ (see Figure S7 for the high-temperature dependence). For all sample, $\kappa(T)$ is characterized by a small but discernible Umklapp peak near $18 \mathrm{~K}$ typical of crystalline solids. Upon warming to $300 \mathrm{~K}, \kappa$ decreases below $1.0 \mathrm{~W} \mathrm{~m}^{-1} \mathrm{~K}^{-1}$ for substitution levels higher than $x=1.2$ with a minimum value of $0.8 \mathrm{~W} \mathrm{~m}^{-1} \mathrm{~K}^{-1}$ achieved at $300 \mathrm{~K}$ in the $x=1.6$ sample. As usually observed in tetradymite compounds, ${ }^{2}$ the anisotropy in $\kappa$ is reversed compared to $\rho$, with an anisotropy ratio $\kappa_{\|} / \kappa_{\perp}$ on the order of 0.75 over the entire temperature and $x$ ranges (Figures S6a and S6b in Supporting Information). However, this anisotropy ratio is not strictly equivalent to the inverse of those observed in the $\rho$ values.

The lattice thermal conductivity $\kappa_{L}$ was estimated by subtracting the electronic contribution $\kappa_{e}=L T / \rho$ to $\kappa$ (Figure 10b). Across the entire temperature range, the temperature dependence of the Lorenz number $L$ was estimated using the aforementioned SPB model. With increasing the Sb content, the magnitude of the Umklapp peak, reaching $1.40 \mathrm{~W} \mathrm{~m}^{-1} \mathrm{~K}^{-1}$ in the $x=0.0$ compound, decreases to $1.15 \mathrm{~W} \mathrm{~m}^{-1} \mathrm{~K}^{-1}$ for $x=2.4$, due to the enhanced disorder induced by this substitution. Upon warming, the $\kappa_{L}$ values tend to a similar, very low value of about $0.6 \mathrm{~W} \mathrm{~m}^{-1} \mathrm{~K}^{-1}$ at $300 \mathrm{~K}$, which only weakly depends on the Sb content. Such a low value is similar to those observed at room temperature in the related polycrystalline compounds $\mathrm{BiSe}$ and $\mathrm{Bi}_{8} \mathrm{Se}_{9} .{ }^{39,40}$ This value is close to the minimum thermal conductivity of $0.38 \mathrm{~W} \mathrm{~m}^{-1} \mathrm{~K}^{-1}$ estimated from room-temperature measurements of the longitudinal $\left(v_{L}=2686 \mathrm{~m} \mathrm{~s}^{-1}\right)$ and transverse $\left(v_{T}=1845 \mathrm{~m} \mathrm{~s}^{-1}\right)$ sound velocities following the high-temperature limit of the formula proposed by Cahill and Pohl. ${ }^{65}$

The nearly equivalent $\kappa_{L}$ values inferred in BiSe (Ref. 39), $\mathrm{Bi}_{8} \mathrm{Se}_{9}$ (Ref. 40) and $\mathrm{Bi}_{8} \mathrm{Se}_{7}$ (Ref. 41) suggest that the stacking sequence of the $\mathrm{Bi}_{2}$ and $\mathrm{Bi}_{2} \mathrm{Se}_{3}$ layers plays a minor role in 
determining the heat transport in this series. This observation contrasts with the results reported for the homologous series $\left(\mathrm{PbSe}_{5}\left(\mathrm{Bi}_{2} \mathrm{Se}_{3}\right)_{3 m}\right.$ with $m=1,2$ and 3 for which, ${ }^{62,66}$ increasing the number of $\mathrm{Bi}_{2} \mathrm{Se}_{3}$ layers $m$ results in an increase in the $\kappa_{L}$ values, which would be expected to tend to those of pure $\mathrm{Bi}_{2} \mathrm{Se}_{3}$ for higher $m$ values. These two distinct behaviors highlight the prominent role played by the layer interfaces that act as an efficient source of phonon diffusion in heterostructures, depending on the lattice mismatch and misalignment of lattice planes between different types of layers.

The inherently low $\kappa_{L}$ values of these layered compounds primarily originates from the large number of atoms per unit cell, giving rise to a high number of dispersionless, optical phonon modes that only weakly contribute to the heat transport. Another important ingredient that contributes to explain the common $\kappa_{L}$ values of these three compounds is the beneficial role played by the $\mathrm{Bi}_{2}$ layers in shaping the low-energy part of the phonon spectrum, as evidenced by lattice-dynamics calculations performed for BiSe. ${ }^{39}$ The thermal motion of the $\mathrm{Bi}$ atoms within the $\mathrm{Bi}_{2}$ layers are associated with low-energy optical modes that strongly limit the phase space available for acoustic phonons and tend to lower the sound velocities. Additional calculations of the mode Grüneisen parameters $\gamma_{q}$, a measure of the bonding anharmonicity, evidenced $\gamma_{q}$ values of the low-energy optical modes exceeding 2, a signature of strong anharmonic behavior. The presence of these $\mathrm{Bi}_{2}$ layers in $\mathrm{Bi}_{8} \mathrm{Se}_{7}$ and $\mathrm{Bi}_{8} \mathrm{Se}_{9}$ likely gives rise to similar features in the low-energy phonon spectrum, explaining the equivalent sound velocities and $\kappa_{L}$ values measured in $\mathrm{BiSe}, \mathrm{Bi}_{8} \mathrm{Se}_{9}$ and $\mathrm{Bi}_{8} \mathrm{Se}_{7}$ despite their different stacking sequences. 


\subsection{Dimensionless Thermoelectric Figure of Merit ZT}

From the combination of the three transport coefficients discussed above, the temperature dependence of the $Z T$ values were inferred. In agreement with prior results, ${ }^{41}$ a maximum $Z T$ value of 0.40 was obtained in the $x=1.6$ sample at $320 \mathrm{~K}$. Note that high-temperature measurements (Figure S8 in ESI) show that the peak $Z T$ value near $320 \mathrm{~K}$ is 0.45 , the difference between the low- and high-temperature data sets being ascribed to the thermal radiations that accompany the low-temperature thermal conductivity measurements. Because the anisotropy in the $\rho$ values is not entirely compensated by the anisotropy in $\kappa$, the $Z T$ values are anisotropic in the entire temperature range (Figures S9 in ESI). Of note, this ZT value is significantly higher than those achieved at room temperature in $n$-type pristine $\mathrm{Bi}_{8} \mathrm{Se}_{9}$ $(0.15 \text { at } 300 \mathrm{~K})^{40}$ or $\mathrm{Bi}_{2} \mathrm{Se}_{3}$ (Ref. 2) and similar to those measured in Sb-substituted BiSe. ${ }^{39}$ This peak $Z T$ obtained in the $x=1.6$ sample is also comparable to those measured in the $n$ type compounds MgAgSb (Refs. 21 and 22) or in doped single- or polycrystalline $\mathrm{Mg}_{3} \mathrm{Sb}_{2}{ }^{24,67-69}$

\section{Conclusion}

We reported on a detailed investigation of the evolution of the low-temperature transport properties of the series of polycrystalline compounds $\mathrm{Bi}_{8-x} \mathrm{Sb}_{x} \mathrm{Se}$. All the synthesized samples crystallize with the trigonal structure of the binary $\mathrm{Bi}_{8} \mathrm{Se}_{7}$ compound described in the space group $P \overline{3} m 1$. As the binary $\mathrm{BiSe}, \mathrm{Bi}_{8} \mathrm{Se}_{7}$ behaves as a narrow-band-gap degenerate $n$-type semiconductor with relatively low thermopower and electrical resistivity values. Both transport coefficients evolve concomitantly upon introducing isovalent $\mathrm{Sb}$ as a result of the electron concentration that decreases with increasing $x$, pointing to a prominent role played by 
native defects. Of note, the variations in both coefficients with $x$ suggests that two distinct substitutional ranges exist in this series, delineated by a concentration located between $x=1.2$ and 1.6. At the optimum doping level of $x=1.6$, a remarkable peak $Z T$ value of 0.40 at $300 \mathrm{~K}$ is achieved, which ranks this compound among the best known $n$-type thermoelectric materials for near-room-temperature applications. Central to these high performances is the poor ability of this layered crystal structure to conduct heat, should it be along or perpendicular to the pressing direction. The stacking sequence of the layers in $\mathrm{Bi}_{8} \mathrm{Se}_{7}$ likely reduces the energy window of the acoustic phonon branches due to the presence of lowenergy optical modes related to the $\mathrm{Bi}_{2}$ layers, resulting in the low sound velocities measured experimentally. The observation of high thermoelectric performances in these $n$-type compounds indicates that the study of the transport properties of other compounds in various tetradymite homologous series may unveil novel materials with superior thermoelectric properties near room temperature upon proper optimization.

\section{Electronic Supplementary Information (ESI) available.}

\section{Conflict of Interest}

The authors declare no conflict of interest. 


\section{References}

${ }^{1}$ H. J. Goldsmid, Thermoelectric Refrigeration, Springer, New York, USA, 1964.

${ }^{2}$ Thermoelectrics and its Energy Harvesting, ed. D. M. Rowe, CRC Press, Boca Raton, FL, 2012.

${ }^{3}$ Y. Pei, X. Shi, A. LaLonde, H. Wang, L. Chen and G. J. Snyder, Nature, 2011, 473, 66-69.

${ }^{4}$ J. Zhang, R. Liu, N. Cheng, Y. Zhang, J. Yang, C. Uher, X. Shi, L. Chen and W. Zhang, Adv. Mater., 2014, 26, 3848-3853.

${ }^{5}$ J. Zhao, C. D. Malliakas, K. Wijayaratne, V. Karlapati, N. Appathurai, D. Y. Chung, S. Rosenkranz, M. G. Kanatzidis and U. Chatterjee, EPL, 2017, 117, 27006.

${ }^{6}$ K. H. Lee, S. Kim, H.-S. Kim and S. W. Kim, ACS Appl. Energy Mater., 2020, 3, 2214 2223.

${ }^{7}$ J. P. Heremans, V. Jovovic, E. S. Toberer, A. Saramat, K. Kurosaki, A. Charoenphakdee, S. Yamanaka and G. J. Snyder, Science, 2008, 321, 554-557.

${ }^{8}$ J. P. Heremans, B. Wiendlocha and A. M. Chamoire, Energy Environ. Sci. 2012, 5, 55105530.

${ }^{9}$ S. Misra, B. Wiendlocha, J. Tobola, F. Fesquet, A. Dauscher, B. Lenoir and C. Candolfi, J. Mater. Chem. C, 2020, 8, 977-988.

${ }^{10}$ B. Wiendlocha, J.-B. Vaney, C. Candolfi, A. Dauscher, B. Lenoir and J. Tobola, Phys. Chem. Chem. Phys., 2018, 20, 12948-12957.

${ }^{11}$ S. R. Brown, S. M. Kauzlarich, F. Gascoin and G. J. Snyder, Chem. Mater., 2006, 18, 1873-1877.

${ }^{12}$ R. Al Rahal Al Orabi, P. Gougeon, P. Gall, B. Fontaine, R. Gautier, M. Colin, C. Candolfi, A. Dauscher, J. Hejtmanek, B. Malaman and B. Lenoir, Inorg. Chem., 2014, 53, 11699 11709. 
13 J.-B. Vaney, J. Carreaud, G. Delaizir, A. Pradel, A. Piarristeguy, C. Morin, E. Alleno, J. Monnier, A. P. Gonçalves, C. Candolfi, A. Dauscher and B. Lenoir, Adv. Electron. Mater., $2015,1,1400008$.

${ }^{14}$ M. Samanta, T. Ghosh, S. Chandra and K. Biswas, J. Mater. Chem. A, 2020, 8, 12226-1 2261.

${ }^{15}$ C. Bourgès, Y. Bouyrie, A. R. Supka, R. Al Rahal Al Orabi, P. Lemoinr, O. I. Lebedev, M. Ohta, K. Suekuni, V. Nassif, V. Hardy, R. Daou, Y. Miyazaki, M. Fornari and E. Guilmeau, J. Am. Chem. Soc., 2018, 140, 2186-2195.

${ }^{16}$ Y. Bouyrie, C. Candolfi, A. Dauscher, B. Malaman and B. Lenoir, Chem. Mater., 2015, 27, $8354-8361$.

${ }^{17}$ J.-B. Labégorre, A. Virfeu, A. Bourhim, H. Willeman, T. Barbier, F. Appert, J. Juraszek, B. Malaman, A. Huguenot, R. Gautier, V. Nassif, P. Lemoine, C. Prestipino, E. Elkaim, L. Poutrot-d'Alençon, T. Le Mercier, A. Maignan and R. Al Rahal Al Orabi, E. Guilmeau, Adv. Funct. Mater., 2019, 29, 1904112.

18 T. Tanimoto, K. Suekuni, T. Tanishita, H. Usui, T. Tadano, T. Kamei, H. Saito, H. Nishiate, C. H. Lee, K. Kuroki and M. Ohtaki, Adv. Funct. Mater., 2020, 30, 2000973.

${ }^{19}$ X. Lu, D. T. Morelli, Y. Xia, F. Zhou, V. Ozolins, H. Chi, X. Zhou and C. Uher, Adv. Energy Mater., 2012, 3, 342-348.

${ }^{20}$ J. Fulmer, O. I. Lebedev, V. V. Roddatis, D. C. Kaseman, S. Sen, J.-A. Dolyniuk, K. Lee, A. V. Olenev and K. Kovnir, J. Am. Chem. Soc., 2013, 135, 12313-12323.

${ }^{21}$ H. Zhao, J. Sui, Z. Tang, Y. Lan, Q. Jie, D. Kraemer, K. McEnaney, A. Guloy, G. Chen and Z. Ren, Nano Energy, 2014, 7, 97-103.

${ }^{22}$ D. Kraemer, J. Sui, K. McEnaney, H. Zhao, Q. Jie, Z. F. Ren and G. Chen, Energy Environ. Sci., 2015, 8, 1299-1308. 
${ }^{23}$ J. Mao, H. Zhu, Z. Ding, Z. Liu, G. A. Gamage, G. Chen and Z. Ren, Science, 2019, 365, 495-498.

${ }^{24}$ H. Tamaki, H. K. Sato and T. Kanno, Adv. Mater., 2016, 28, 10182-10187.

25 J. Zhang, L. Song, S. H. Pedersen, H. Yin, L. T. Hung and B. B. Iversen, Nat. Commun., $2017, \mathbf{8}, 13901$.

${ }^{26}$ A. Mrotzek and M. G. Kanatzidis, Acc. Chem. Res., 2003, 36, 111-119.

${ }^{27}$ B. Makovicky, W. C. Mumme and J. A. Watts, Can. Mineral., 1977, 15, 339-348.

${ }^{28}$ A. Olvera, G. Shi, H. Djieutedjeu, A. Page, C. Uher, E. Kioupakis and P. F. P. Poudeu, Inorg. Chem. 2015, 54, 746-755.

${ }^{29}$ J. Casamento, J. S. Lopez, N. A. Moroz, A. Olvera, H. Djieutedjeu, A. Page, C. Uher and P. F. P. Poudeu, Inorg. Chem., 2017, 56, 261-268.

${ }^{30}$ M. Ohta, D. Y. Chung, M. Kunii and M. G. Kanatzidis, J. Mater. Chem. A, 2014, 2, 20048 20058.

${ }^{31}$ A. Banik and K. Biswas, Angew.Chem. Int. Ed., 2017, 56,14561 -14566.

${ }^{32}$ S. Sassi, C. Candolfi, V. Ohorodniichuk, C. Gendarme, P. Masschelein, A. Dauscher and B. Lenoir, J. Electron. Mater., 2017, 46, 2790-2796.

${ }^{33}$ S. Sassi, C. Candolfi, C. Gendarme, A. Dauscher and B. Lenoir, Dalton Trans., 2018, 47, $4714-4721$.

${ }^{34}$ A. A. Sher, I. P. Odin and A. V. Novoselova, Zhurnal Neorganicheskoi Khimii, 1986, 31, 435.

${ }^{35}$ R. F. Brebrick, J. Appl. Cryst., 1968, 1, 241-246.

${ }^{36}$ Y. Feutelais, B. Legendre, N. Rodier and V. Agafonov, Mat. Res. Bull., 1993, 28, 591-596.

${ }^{37}$ H. Lind and S. Lidin, Solid State Sci., 2003, 5, 47-57.

${ }^{38}$ H. Lind, S. Lidin and U. Häussermann, Phys. Rev. B, 2005, 72, 184101. 
${ }^{39}$ M. Samanta, K. Pal, P. Pal, U. V. Waghmare and K. Biswas, J. Am. Chem. Soc., 2018, 140, $5866-5872$.

${ }^{40}$ R. Wang, F. Jia, L. Chen and L.-M. Wu, Cryst. Growth Des., 2020, 20, 3555-3560.

${ }^{41}$ F. Jia, Y.-Y. Liu, Y.-F. Zhang, X. Shu, L. Chen and L.-M. Wu, J. Am. Chem. Soc., 2020, 142, 12536-12543.

${ }^{42}$ H. Okamoto, JPE, 1994, 15, 195-201.

${ }^{43}$ J. Rodriguez-Carvajal, Physica B, 1993, 192, 55-69.

${ }^{44}$ P. Kubelka and F. Z. Munk, Technol. Phys., 1932, 12, 593-601.

${ }^{45}$ Y. C. Akgöz and G. A. Saunders, J. Phys. C: Solid State Phys., 1975, 8, 1387-1396.

${ }^{46}$ E. Alleno, D. Bérardan, C. Byl, C. Candolfi, R. Daou, R. Decourt, E. Guilmeau, S. Hébert, J. Hejtmanek, B. Lenoir, P. Masschelein, V. Ohorodniichuk, M. Pollet, S. Populoh, D. Ravot, O. Rouleau, M. Soulier, Rev. Sci. Instrum., 2015, 86, 011301.

${ }^{47}$ M. M. Stasova and O. G. Karpinskii, Zhurnal Strukturnoi Khimii, 1967, 8, 85-88.

${ }^{48}$ J. W. G. Bos, H. W. Zandbergen, M.-H. Lee, N. P. Ong and R. J. Cava, Phys. Rev. B, 2007, 75, 195203.

49 T. Zhang, Y. Jiang, Z. Song, H. Huang, Y. He, Z. Fang, H. Weng and C. Fang, Nature, 2019, 566, 475-479.

${ }^{50}$ M. Samanta, K. Pal, U. V. Waghmare and K. Biswas, Angew. Chem. Int. Ed., 2020, 59,4822-4829.

51 J. Horák, Z. Stary, P. Losták and J. Pancír, J. Phys. Chem. Solids, 1990, 51, 1353-1360.

52 J.-P. Issi, Aust. J. Phys. 1979, 32, 585-628.

${ }^{53}$ H. J. Goldsmid and J. W. Sharp, J. Electron. Mater., 1999, 28, 869-872.

${ }^{54}$ V. G. Kuznetsov, K. K. Palkina and A. A. Reshchikova, Inorg. Mater., 1968, 4, 670-677.

${ }^{55}$ C. Drasar, I. Klichova, L. Koudelka and P. Losták, Cryst. Res. Technol., 1996, 31, 805-812. 
56 V. A. Kulbachinskii, N. Miura, H. Nakagawa, H. Arimoto, T. Ikaida, P. Lostak and C. Drasar, Phys. Rev. B, 1999, 59, 15733.

${ }^{57}$ D. West, Y. Y. Sun, W. Han, B. Junhyeok and S. B. Zhang, Phys. Rev. B, 2012, 86, 121201.

${ }^{58}$ L. Xue, P. Zhou, C. X. Zhang, C. Y. He, G. L. Hao, L. Z. Sun and J. X. Zhong, AIP Advances, 2013, 3, 052105.

${ }^{59}$ T. Plenchácek, J. Navrátil and J. Horák, J. Solid State Chem., 2002, 165, 35-41.

${ }^{60}$ T. R. Devidas, E. P. Amaladass, S. Sharma, A. Mani, R. Rajaraman, C. S. Sundar and A. Bharathi, Mater. Res. Express, 2017, 4, 026101.

${ }^{61}$ T. R. Devidas, E. P. Amaladass, S. Sharma, R. Rajaraman, D. Sornadurai, N. Subramanian, A. Mani, C. S. Sundar and A. Bharathi, EPL, 2014, 108, 67008.

${ }^{62}$ S. Sassi, C. Candolfi, G. Delaizir, S. Migot, J. Ghanbaja, C. Gendarme, A. Dauscher, B. Malaman and B. Lenoir, Inorg. Chem., 2018, 57, 422-434.

${ }^{63}$ S. Ohno, U. Aydemir, M. Amsler, J.-H. Pöhls, S. Chanakian, A. Zevalkink, M. A. White, S. K. Bux, C. Wolverton and G. J. Snyder, Adv. Funct. Mater., 2017, 27, 1606361.

${ }^{64}$ V. I. Fistul in Heavily Doped Semiconductors, Plenum Press, New York, 1969.

${ }^{65}$ D. G. Cahill, S. K. Watson and R. O. Pohl, Phys. Rev. B, 1992, 46, 6131-6140.

${ }^{66}$ S. Sassi, C. Candolfi, A. Dauscher, B. Lenoir and M. M. Koza, Phys. Chem. Chem. Phys., 2018, 20, 14597-14607.

${ }^{67}$ K. Imasato, S. D. Kang and G. J. Snyder, Energy Environ. Sci., 2019, 12, 965-971.

${ }^{68}$ X. Shi, X. Wang, L. Wen and Y. Pei, Small Methods, 2018, 2, 1800022.

${ }^{69}$ Y. Pan, M. Yao, X. Hong, Y. Zhu, F. Fan, K. Imasato, Y. He, C. Hess, J. Fink, J. Yang, B. Büchner, C. Fu, G. J. Snyder and C. Felser, Energy Environ. Sci., 2020, 13, 1717-1724. 


\section{Figure Captions}

Figure 1. Crystal structure of $\mathrm{Bi}_{8} \mathrm{Se}_{7}$ viewed along the [110] direction showing the stacking sequence 2525525255252 of alternating Bi2 layers formed by Bi zigzag chains (denoted as '2') and $\mathrm{Bi}_{2} \mathrm{Se}_{3}$ quintuple layers (denoted as '5'). Bi and Se atoms are shown in purple and light green, respectively. The twelve and eleven independent crystallographic sites over which, Bi and Se are distributed, respectively, are not distinguished. The edge-sharing chains of $\mathrm{BiSe}_{6}$ octahedra within the quintuple layers are shown in light blue. The solid black lines represent the unit cell.

Figure 2. a) Powder X-ray diffraction patterns for the series $\mathrm{Bi}_{8-x} \mathrm{Sb}_{x} \mathrm{Se}_{7}(0.0 \leq x \leq 2.4)$. Selected reflections are indexed based on the simulated PXRD pattern shown at the bottom. b) Magnification of the PXRD patterns around the reflection near $2 \theta=40^{\circ}$ to highlight the systematic shift upon increasing the Sb content.

Figure 3. a) Unit cell volume $V$ as a function of the Sb concentration $x$. The solid black line stands for the best linear fit to the data. b) Lattice parameters $a$ and $c$ as a function of the $\mathrm{Sb}$ concentration $x$ inferred from profile matching of the PXRD patterns. The black solid lines stand for the best linear fit to the data. The error bars for $a$ are smaller than the size of the symbols used.

Figure 4. Backscattered electron (BSE) images and corresponding elemental X-ray maps of the illustrative $x=1.6$ sample. 
Figure 5. SEM images at different magnifications of the microstructure of the illustrative $x=$ 0.0 sample taken on a fresh fracture of a consolidated polycrystalline piece. Two magnifications areas (panels $b$ and $c$ ) of the overall overview shown in panel a show the stackings of long plate-like grains, with the sheets (highlighted in panel d) tending to stack along the SPS direction.

Figure 6. Optical absorption spectra showing the normalized Kubelka-Munk function $F(R)$ as a function of the photon energy $\hbar \omega$ (in $\mathrm{eV}$ ) for the $x=0.0$ (red filled circles), 1.2 (blue filled squares) and 2.4 (green filled upper triangle) samples. The two observed transitions are indicated by the black solid lines.

Figure 7. Temperature dependence of a) the electrical resistivity $\rho$, b) the thermopower $\alpha$, and c) the power factor $\alpha^{2} / \rho$ for the series $\mathrm{Bi}_{8-x} \mathrm{Sb}_{x} \mathrm{Se}_{7}(0.0 \leq x \leq 2.4)$. The color-coded symbols are similar in the three panels.

Figure 8. Temperature dependence of a) the Hall electron concentration $n_{H}$ and b) the Hall mobility $\mu_{H}$ for the series $\mathrm{Bi}_{8-x} \mathrm{Sb}_{x} \mathrm{Se}_{7}(0.0 \leq x \leq 2.4)$. The $T^{-3 / 2}$ law represented by the solid black line in panel $\mathrm{b}$ corresponds to the temperature dependence expected for acoustic phonon scattering.

Figure 9. Ioffe-Pisarenko plot at $50 \mathrm{~K}$ showing the thermopower $\alpha$ (in absolute values) as a function of the Hall electron concentration $n_{H}$. The two concentration ranges $0.0 \leq x \leq 1.2$ and $1.6 \leq x \leq 2.4$ are distinguished by blue filled square and red filled circle symbols, respectively. The solid and dashed curves were calculated by a single-parabolic band model with neutral 
impurity scattering. The error bars correspond to experimental uncertainties of $5 \%$ for both $\alpha$ and $n_{H}$

Figure 10. Temperature dependence of a) the total thermal conductivity $\kappa$ and b) the lattice thermal conductivity $\kappa_{L}$ for the series $\mathrm{Bi}_{8-x} \mathrm{Sb}_{x} \mathrm{Se}_{7}(0.0 \leq x \leq 2.4)$. The color-coded symbols are similar in both panels.

Figure 11. Temperature dependence of the dimensionless thermoelectric figure of merit $Z T$ for the series $\mathrm{Bi}_{8-x} \mathrm{Sb}_{x} \mathrm{Se}_{7}(0.0 \leq x \leq 2.4)$. 


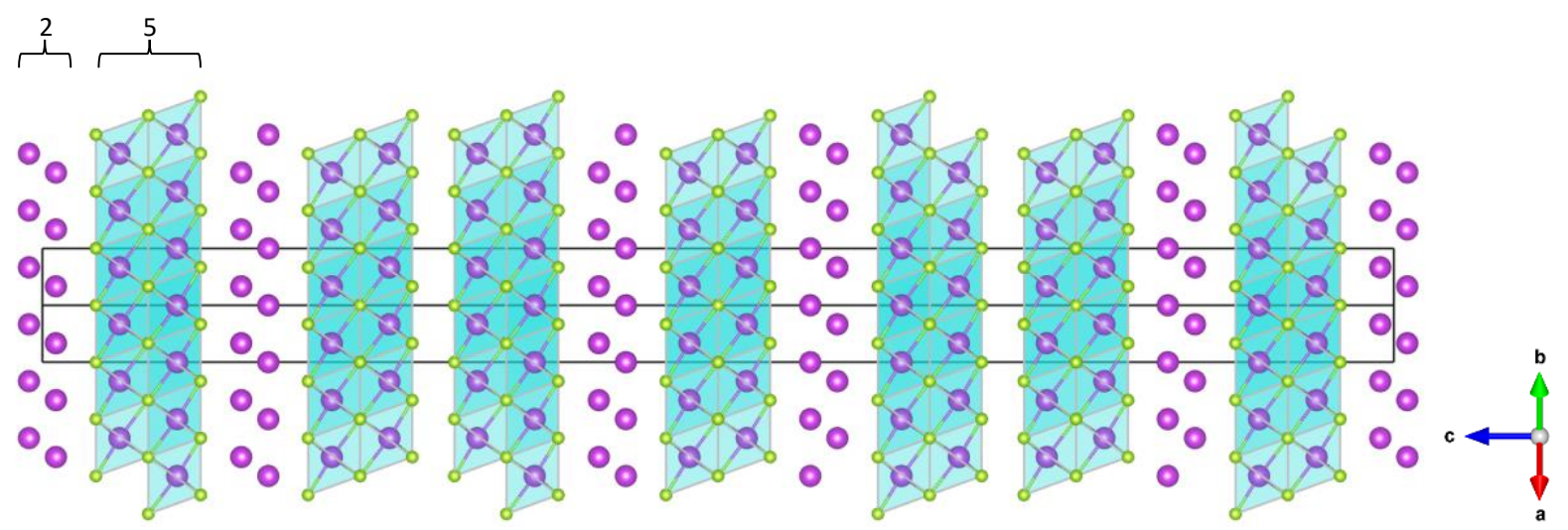

Figure 1 
(a)

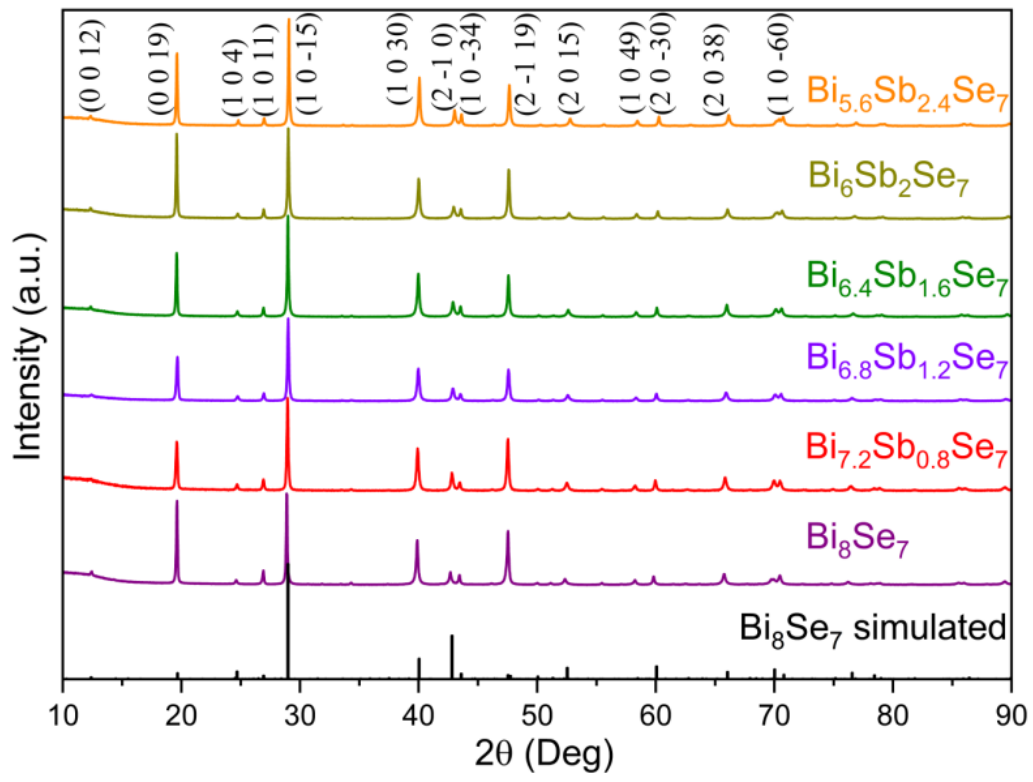

(b)

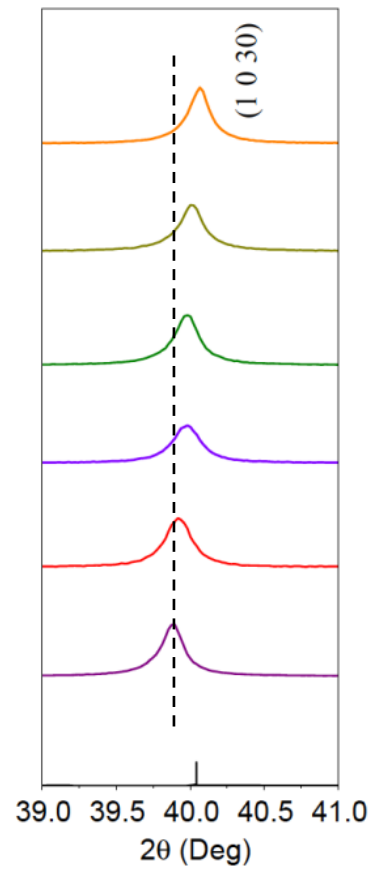

Figure 2 

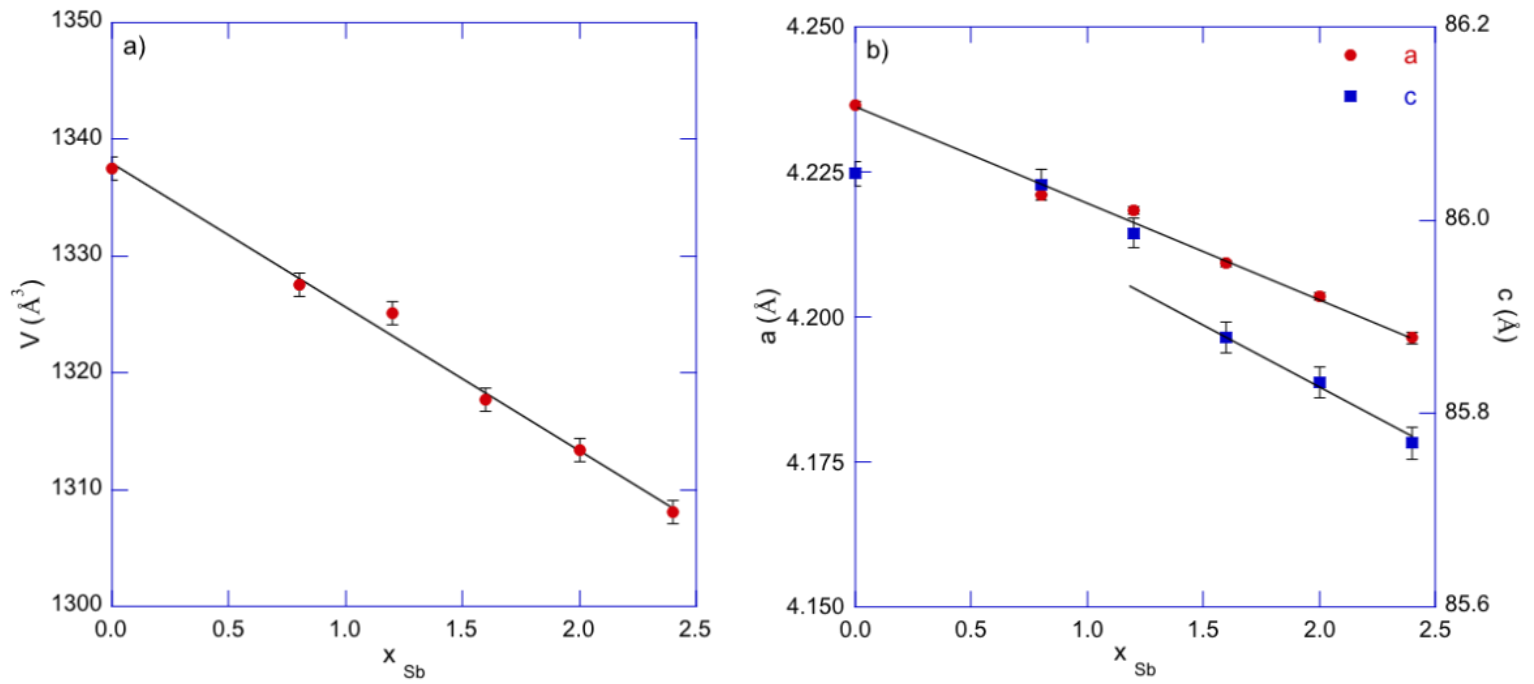

Figure 3 

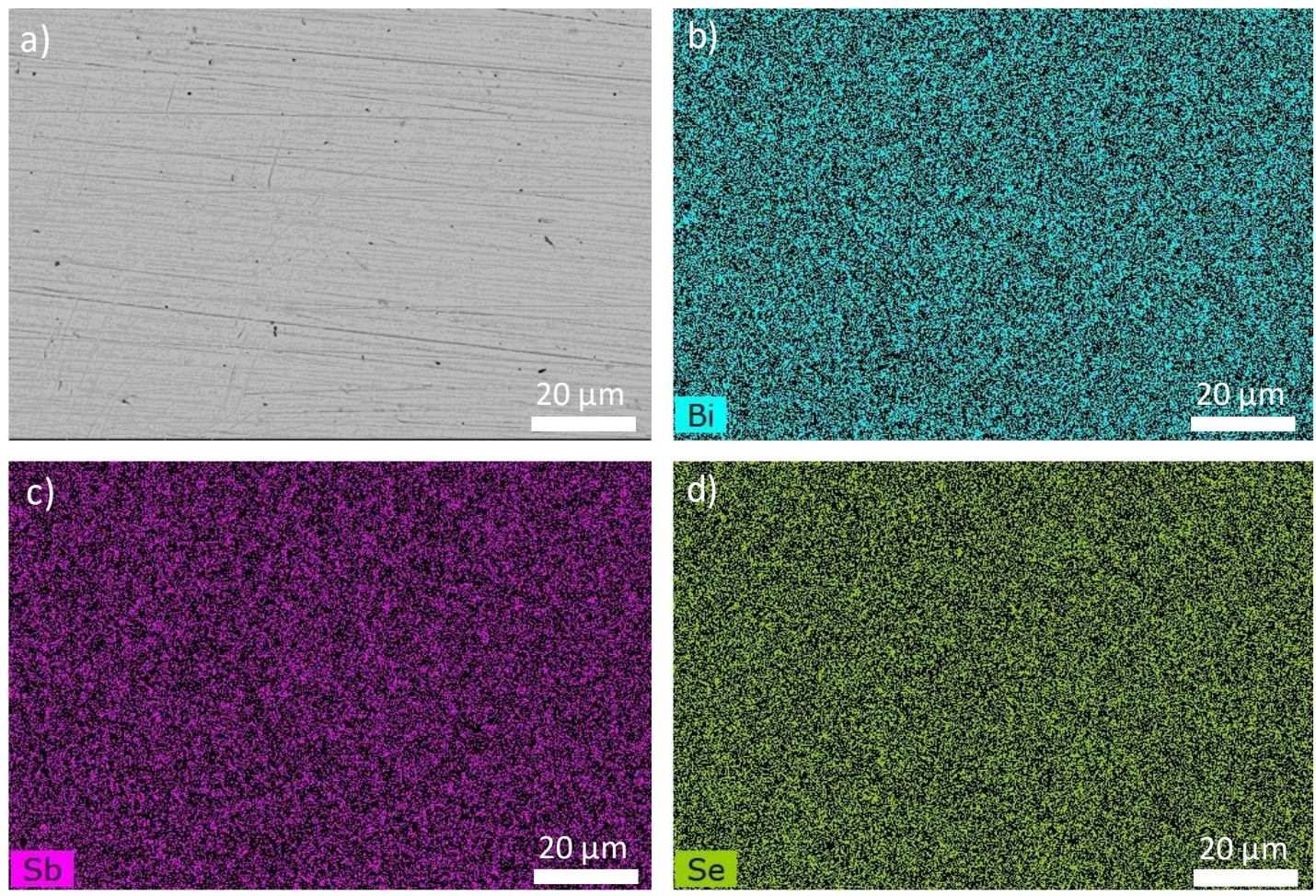

Figure 4 

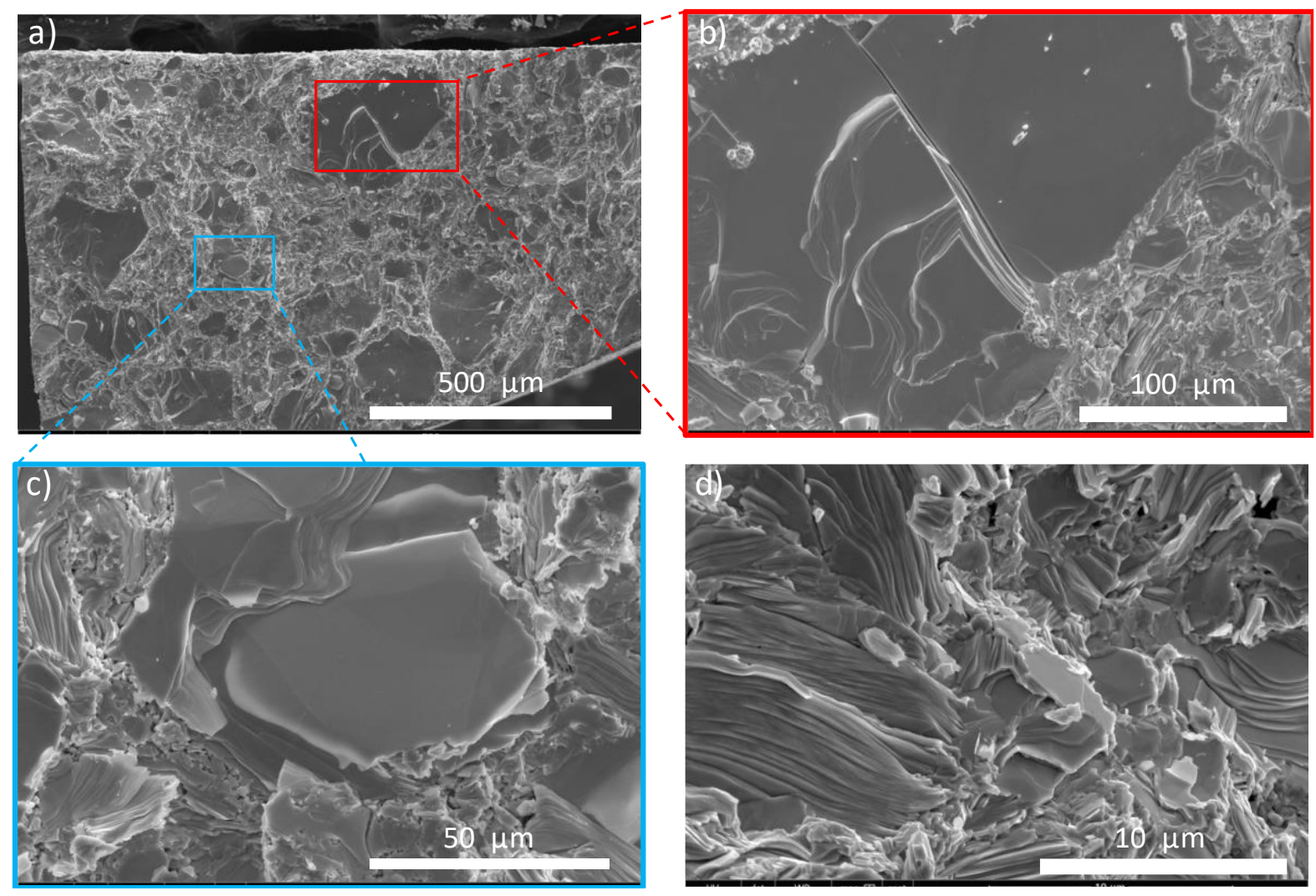

Figure 5 


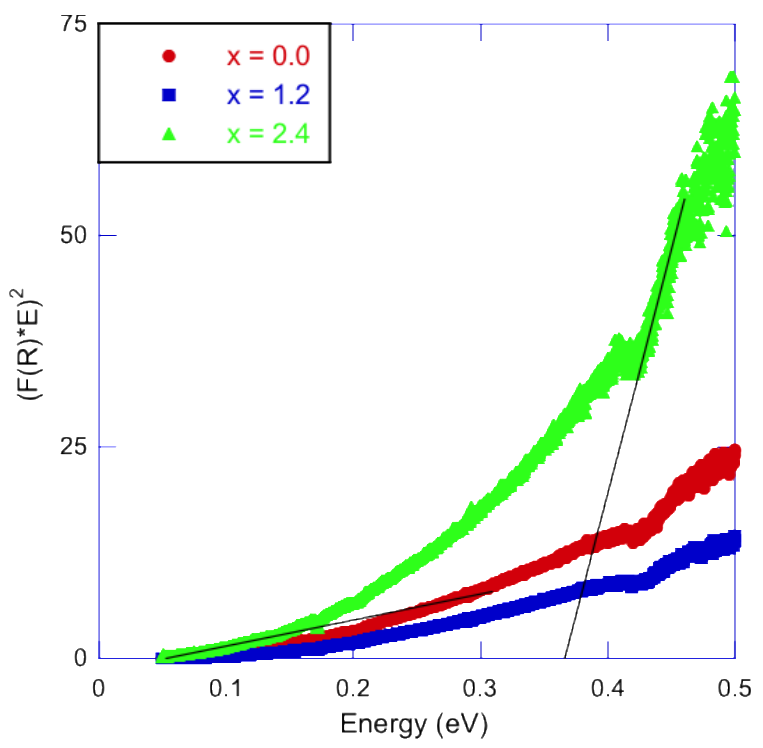

Figure 6 

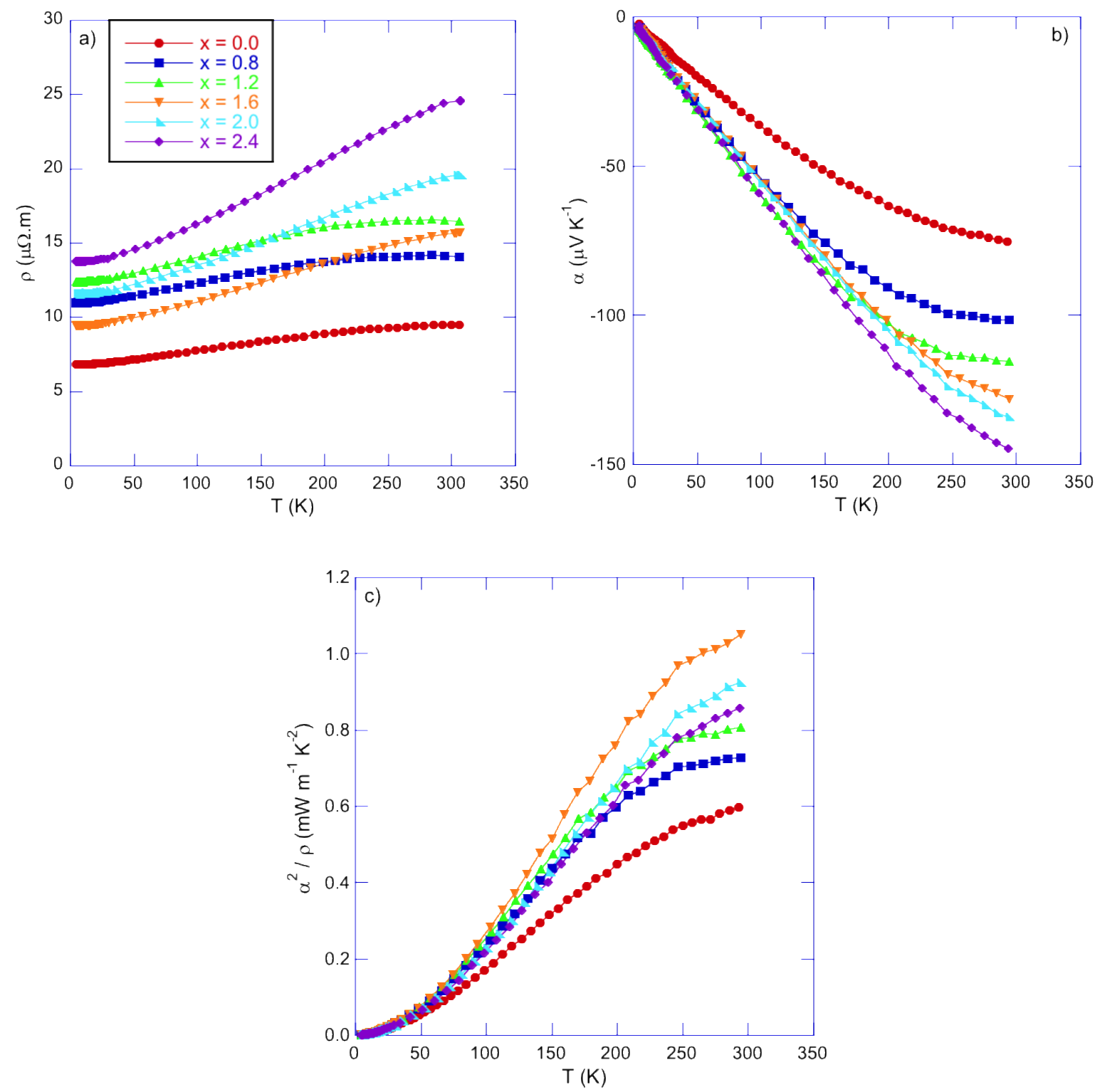

Figure 7 

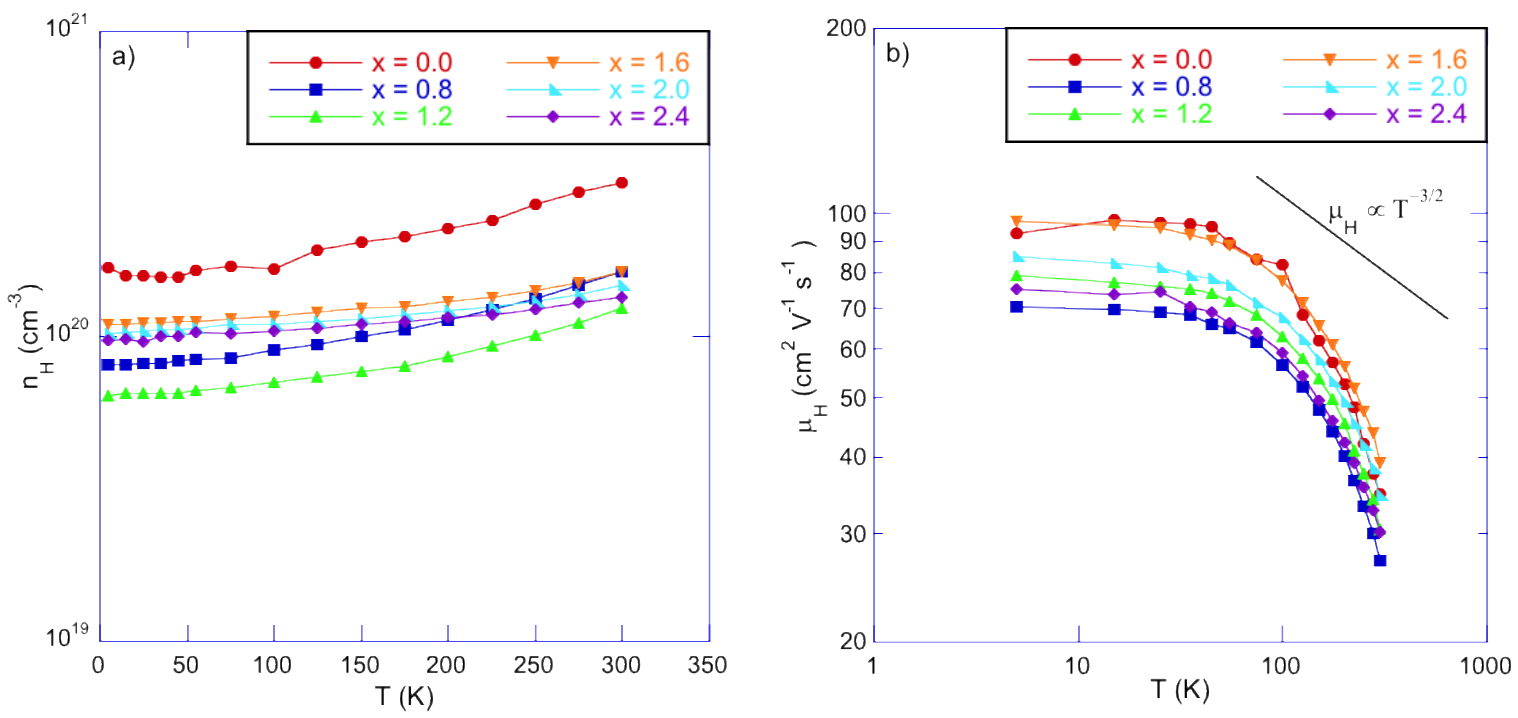

Figure 8 


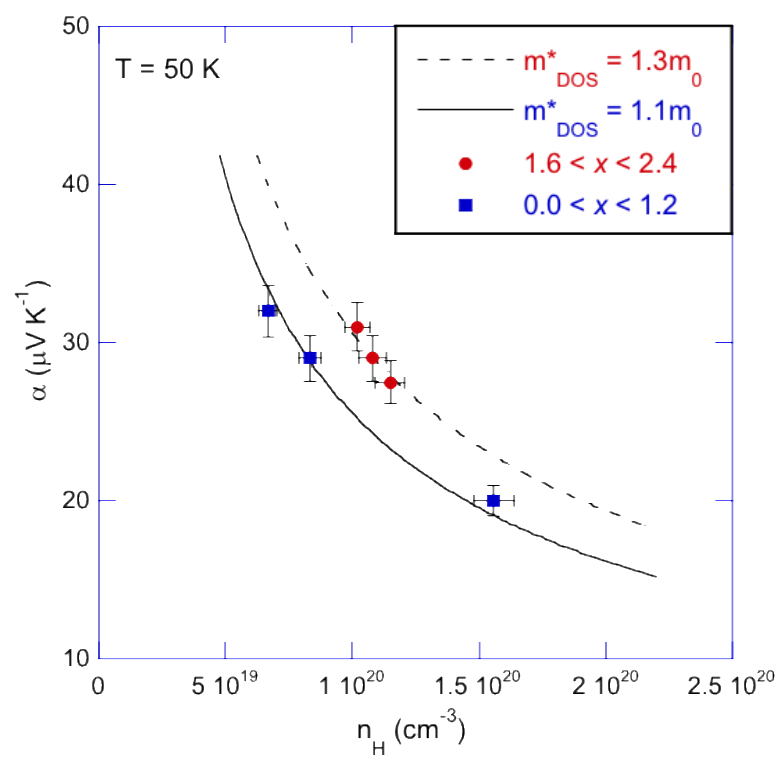

Figure 9 

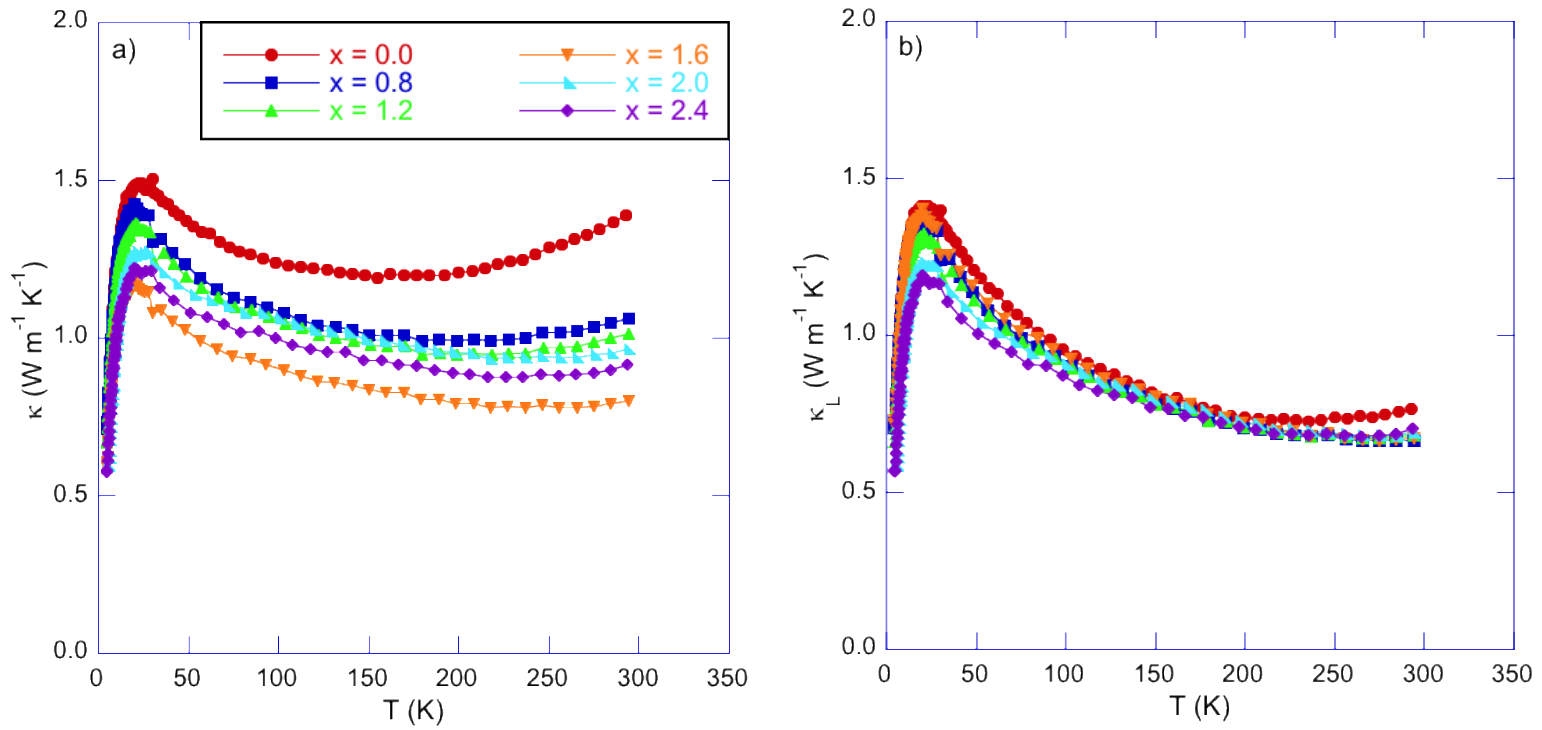

Figure 10 


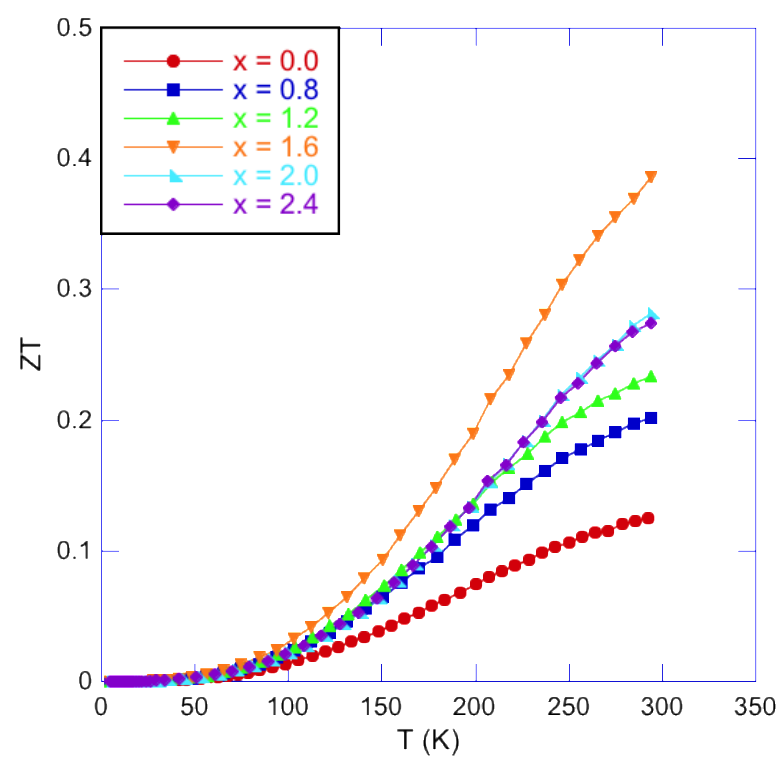

Figure 11 